Commentary

\title{
Sensational developments in the irritable bowel
}

Since generation of the hypothesis that chronic visceral hyperalgesia may play an important part in symptom causation in functional gastrointestinal disorders (FGD), remarkable progress has been made in some areas of research into these complicated problems. For example, computerised devices are now being used to deliver defined distension stimuli to the gut, candidate drugs aimed at decreasing enhanced sensitivity to visceral events are being tested in phase II trials, and a whole range of response parameters from subjective sensations to autonomic responses and regional blood flow changes in the brain are being evaluated. On the other hand, even the most recent published sensory FGD studies have not paid enough attention to well established lessons from psychophysics (the scientific study of perception). There is still not a single published report on altered visceral sensitivity in FGD patients that has used sufficiently stringent pyschophysical measures to allow us to identify the precise nature of the perceptual alteration(s) in these patients. Are we dealing with allodynia (experience of normally innocuous stimuli as painful), dysesthesia (experience of normally innocuous stimuli as unpleasant), hyperalgesia (lowered threshold for painful stimuli), hypervigilance (an increased focus and response to specific negative sensations), or other forms of response bias (in which non-sensory cues influence the rating of stimulus intensity)? As long as we don't know what the underlying problem is, it is difficult to extrapolate to underlying mechanisms, and it is even more difficult to interpret postulated results of pharmacological interventions. For example, is the therapeutic effect of octreotide on rectal sensitivity in irritable bowel syndrome patients a result of reduced rectal compliance in these patients or a direct effect on afferent pathways? ${ }^{1}$

The interesting study by Louvel et al in this issue illustrates both the progress and the continuing problems that characterise current studies (including our own!) on the perception of experimental gastrointestinal distension. The Toulouse group, which has extensive experience with visceral distension studies, reports that parenteral oxytocin in doses of $20 \mathrm{mU}$ and greater increases the distension pressure at which irritable bowel syndrome patients first report the initial perception of the distending stimulus, and the pressure at which they first report pain. This oxytocin effect was not affected by naloxone and did not seem to be related to drug induced changes in colonic compliance. On the positive side, the authors have used a randomised, placebo controlled protocol to assess the effect of different doses of oxytocin (rarely done in visceral distension studies) on the perceptual response to controlled distension of the descending colon. They have also separately assessed changes in perception and pain threshold. On the negative side, several commonly used features of the distension protocol make the results more difficult to interpret. Firstly, the authors presented their distension stimuli in an ascending series. While this is the most common protocol for assessment of sensory thresholds in the FGD literature, it is highly susceptible to response bias because the subject quickly realises that each stimulus being judged is predictably greater than the previous one. Recent studies by Mertz et $a l^{2}$ and Silverman $e t a l^{3}$ suggest how powerful this response bias can be: patients with functional dyspepsia and even healthy controls during an ascending series rate sham distensions of the stomach (disconnection of the gastric balloon from the distension device during an anticipated distension) very similar to actual distensions. Furthermore, changes in regional cerebral blood flow associated with the perception of high intensity rectal distension are similar to those observed during sham distension. The issue of response bias is particularly problematic in a situation in which the same series of stimuli is being evaluated many times during the experiment (in this case four times over a period of 36 hours) and when the intention is to measure a drug effect on visceral afferent pathways and not presumably psychological expectations. Secondly, the authors used a distending stimulus, which rises rapidly (phasic phase) to an isobaric plateau lasting five minutes (tonic phase) followed by a rapid deflation of the bag (phasic phase). It has been observed by several investigators that subjects will rate threshold and intensity of phasic and tonic stimuli differently, ${ }^{4-6}$ and that pharmacological and other interventions may differentially affect the response to one but not the other type of stimulus. ${ }^{78}$ Depending on when the perceptual response was obtained, the distension stimulus may be considered phasic (initial few seconds) or tonic (towards the end of the isobaric plateau). The length of the protocol illustrates another problem inherent in doing the necessary dose response studies on patients using invasive distension techniques. Randomisation of the order of drug conditions is imperative because the stress and autonomic activity associated with extended balloon insertion will almost certainly affect perceptual responses over the time period of the study. Thus, what we conclude from studies using sensory testing must be tempered by design issues such as those listed above.

Future studies of what may be multiple interacting sensory alterations in FGD need to recognise the complexity of making interpretations of underlying neurophysiological processes from subjective perceptual judgements. At the initial level, sensory studies are influenced by local changes in the gastrointestinal tract, which change how mechanical and chemical stimuli impact on the sensory nerves. As stated above tonic and phasic stimuli are not equivalent and careful specification of stimulus timing and location is critical for comparing results across studies. Although it is surmised that important modulation of visceral sensation takes place in peripheral and spinal nociceptive pathways, this activity can only be studied indirectly through use of compounds that selectively act on spinal neurons, or perhaps by use of conditioning stimuli that facilitate or inhibit nociception at spinal sites, as we and others have done using repetitive sigmoid stimulation. ${ }^{9}{ }^{10}$ Assessment of perceptual alterations that are located in the CNS will involve careful correlation of subjective reports with emerging technologies 
of functional brain imaging (PET and $\mathrm{fMRI}$ ). This process will be greatly aided by the use of sensitive and validated scales of sensory and affective intensity such as those proposed by Gracely et $a l^{11}$ and clear separation of qualitatively different sensory descriptors such as pain and discomfort. A final potential mechanism of altered perception in FGD is that of hypervigilance, or an increased focus on (and increased bias towards) the report of negative somatic or visceral sensations. Given the likelihood that hypervigilance plays an important part in FGD, all sensory studies should use adequate controls for response bias. In typical testing situations this means elimination of non-sensory cues for stimulus intensity such as pump sounds, longer inflation times for greater pressure, etc. In this context, the paradigm for stimulus presentation is also important. Ascending series of stimuli are highly prone to bias both due to predictability and fear of injury. ${ }^{12}$ Thresholds determined from random stimulus presentations or tracking protocols, in which the order of the stimuli is unavailable to the subject, lead to changes in judgments based more on sensory properties than changes in labels for the same sensation. ${ }^{13}$ Analyses based on sensory decision theory, which use subjects' response consistency to derive independent measures of response bias and discrimination ability may also prove useful, although the validity of these indices when applied to pain measurement has been questioned. ${ }^{14-16}$ Finally, threshold judgments, even if unbiased, may not sufficiently represent sensitivity across the full stimulus range of interest. As clinical practice usually entails management of moderate to intense discomfort, it is important in sensory protocols to assess suprathreshold sensation in addition to that of pain or discomfort threshold. Findings from a factor analysis of sensory testing data in our laboratory provides empirical evidence for at least two relatively distinct perceptual alterations associated with irritable bowel syndrome. The first and most common might be labelled hypervigilance for visceral sensations, reflected in an earlier use of the discriptor discomfort during an ascending series of rectal stimuli, and a lower tolerance during the same series. A second factor, visceral sensitivity, is composed of the discomfort threshold assessed during a non-biased tracking paradigm and verbal descriptor ratings of suprathreshold stimuli. ${ }^{17}$
The use of experimental sensory testing protocols holds immense promise for our understanding of functional gastrointestinal disorders. Careful design of future studies should allow for testing of specific hypotheses regarding the mechanism of dysregulation in these disorders and the site of action of new intervention strategies.

BRUCE NALIBOFF EMERAN A MAYER CURE Digestive Diseases Research Center/Neuroenteric Biology Group
UCLA, Los Angeles, CA 90073 and WLA-VA Medical Center, USA

1 Bradette M, Delvaux M, Staumont G, Fioramonti J, Bueno L, Frexinos J. Octroetide increases thresholds of colonic visceral perception in IBS patients without modifying muscle tone. Dig Dis Sci 1994; 39: 1171-8.

2 Mertz H, Fass R, Hirsh T, Yan-Go F, Mayer EA. Amitryptiline for functional dyspepsia: effect on symptoms, gastric sensitivity and sleep. Gastroenterology 1995; 108: A649.

3 Silverman DHS, Munakata J, Hoh CK, Mandelkern M, Phelps ME, Blahd W, et al. Regional cerebral activity in normal and pathologic perception of visceral pain: an O-15-water PET study of healthy subjects and IBS patients. Gastroenterology 1996; 110: A759.

4 Sun WM, Read NW, Prior A, Daly J, Cheah SK, Grundy D. The sensory and motor responses to rectal distension vary according to the rate and pattern of balloon inflation. Gastroenterology 1990; 99: 1008-13.

5 Lembo T, Munakata J, Mertz H, et al. Evidence for the hypersensitivity of lumbar splanchnic afferents in irritable bowel syndrome. Gastroenterology 1994; 107: 1686-96.

6 Mertz H, Naliboff B, Munakata J, Niazi N, Mayer E. Altered rectal perception is a biological marker of patients with the irritable bowel syndrome. Gastroenterology 1995; 109: 40-52.

7 Lembo T, Plourde V, Shui Z, et al. Effects of the corticotropin-releasing factor (CRF) on rectal afferent nerves in humans. Neurogastroenterol Motil 1996; 8: $9-18$.

8 Plourde V, Lembo T, Shui Z, et al. Effects of the somatostatin analogue octrotide on rectal afferent nerves in humans. Am $\mathcal{F}$ Physiol 1993; 265: G742-51.

9 Serra J, Azpiroz F, Malagelada J-R. Perception and reflex responses to intestinal distension in humans are modified by simultaneous or previous stimulation. Gastroenterology 1995; 109: 1742-9.

10 Munakata J, Chang L, An C, Naliboff B, Mayer EA. Repetitive activation of sigmoid mechanoreceptors results in the development of rectal hyperalgesia in IBS patients. Gastroenterology 1995; 108: A653.

11 Gracely RH, McGrath P, Dubner R. Validity and sensitivity of ratio scales of sensory and affective verbal pain descriptors: manipulation of affect by diazepam. Pain 1976; 2: 19-29.

12 Gescheider GA. Psychophysics: method, theory, and application. Hillsdale: Lawrence Erlbaum Associates, 1985.

13 Gracely RH. Pain psychophysics. In: Chapman CR, Loeser JD, eds. Advances in pain research and therapy: issues in pain measurement. New York: Advances in pain research and the

14 Rollman GB. Signal detection theory measurement of pain: A review and critique. Pain 1977; 3: 187-211.

15 Chapman CR. Sensory decision theory methods in pain research: A reply to Rollman. Pain 1977; 3: 295-305.

16 Coppola R, Gracely RH. Where is the noise in SDT pain assessment? Pain 1983; 17: 257-66.

17 Naliboff B, Munakata J, Kodner A, Mayer EA. IBS non-patients do not differ from healthy controls on visceral sensitivity. Gastroenterology 1996; 110: A723. 